\title{
Terapia de Aceitação e Compromisso: É uma Proposta de Intervenção Cognitivista?
}

\author{
Terapia de Aceptación y Compromisso: ¿Es una Propuesta de \\ Intervención Cognitivista?
}

\section{Acceptance and Commitment Therapy: Is it a Cognitive Intervention Model?}

\author{
Nazaré Costa ${ }^{1}$
}

[1] Universidade Federal do Maranhão I Título abreviado: Terapia de Aceitação e Compromisso: Cognitivismo? I Endereço para correspondência: Av. dos Holandeses, s/n. Ed. Porto Ravena, apt. 1301. CEP: 65075-650. São Luis-Ma I Email: naza.pc@gmail.com

\begin{abstract}
Resumo: A Terapia de Aceitação e Compromisso (ACT), proposta por Steven Hayes no final da década de 1980, tem sido colocada, frequentemente, inclusive pelo seu fundador, no rol das terapias cognitivas e comportamentais. Distintos podem ser os incômodos com a proposta, com sua fundamentação específica (Teoria dos Quadros Relacionais) e com o próprio Hayes. Entretanto, argumenta-se que, mesmo usando termos evitados por grande parte dos analistas do comportamento, como mente e cognição, por exemplo, a ACT consiste em um modelo terapêutico completamente compatível com o Behaviorismo Radical de Skinner e com a Análise do Comportamento. Entre as concepções filosóficas em comum com o Behaviorismo Radical, destacam-se a visão de comportamento como um fenômeno relacional e a de pensamento/cognição como um evento comportamental, e não causal. Assim, neste artigo busca-se discutir em que medida a ACT pode ser caracterizada como um modelo cognitivista ou apenas comportamental de intervenção.
\end{abstract}

Palavras-chave: Terapia de aceitação e compromisso, modelo comportamental, modelo cognitivista, terapia analítico-comportamental.

\begin{abstract}
The Acceptance and Commitment Therapy (ACT), proposed by Steven Hayes in the late ' 80 s, has been included, often even by its founder, among the cognitive and behavioral therapies. Different can be the discomforts with the proposal, with the specific grounding used (Relational Frame Theory) and Hayes himself. However, it is argued that even using terms which are largely avoided by behavior analysts, as mind and cognition, for example, the ACT consists of a therapeutic model fully compatible with Skinner's Radical Behaviorism and the Behavior Analysis. Among the philosophical ideas in common with the radical behaviorism, we highlight the view behavior as a relational phenomenon and thought/cognition as a behavioral event and not causal. Therefore, this paper seeks to discuss the extent to which ACT can be characterized as a cognitive or only behavioral model of intervention.
\end{abstract}

Key words: Acceptance and commitment therapy, behavioral model, cognitive model, clinical behavioral analysis. 
Resumen: La Terapia de Aceptación y Compromiso (ACT), propuesta por Steven Hayes por los años 1980, se ha colocado, a menudo, incluso por su fundador, entre las terapias cognitiva y conductual. Distintas pueden ser las molestias con la propuesta, con la teoría específica la que se basa (Teoría del Marco Relacional) y con el propio Hayes. Sin embargo, se argumenta aun que la ACT utilize palabras que en gran medida los analistas del comportamiento al menos evitan, como mente y cognición, por ejemplo, la ACT es un modelo terapéutico completamente compatible con el Conductismo Radical de Skinner y con el Análisis Conductual. Entre las ideas filosóficas en común con el Conductismo Radical, se destaca que entiende el comportamiento como un fenómeno relacional y el pensamiento/la cognición como un evento conductual y no causal. Por lo tanto, este artículo pretende analizar el grado em que la ACT se puede caracterizar como un modelo cognitivo o solo conductual de intervención.

Palabras-clave: Terapia de aceptación y compromiso, modelo conductual, modelo cognitivista, análisis clínica conductual. 
O debate acerca da relação entre Cognitivismo e Comportamentalismo e do papel da cognição sobre o comportamento humano não é recente, tampouco se encontra esgotado. Esse debate é evidenciado tanto na literatura internacional (Forsyth, Lejuez, Hawkins \& Eifert, 1996; Hawkins, 1996; Hayes \& Wilson, 1995) quanto na nacional (Barbosa \& Borba, 2010; Costa, 2002; Guilhardi \& Queiroz, s.d). Entretanto, o presente artigo não visa a discorrer acerca das diferenças e possíveis semelhanças entre Cognitivismo e Comportamentalismo ou sobre a possibilidade de integrá-los, nem sobre a função da cognição. Afinal, como afirmaram Hayes e Wilson (1995), discutir sobre o papel causal da cognição não é apenas uma questão empírica. A discussão passa, sobretudo, por concepções acerca dos "objetivos da ciência, natureza da verdade e o status pragmático ou ontológico das causas” (p. 241).

Com este artigo, pretende-se discutir em que medida a Terapia de Aceitação e Compromisso (em inglês, Acceptance and Commitment Therapy - ACT) poderia ser caracterizada como um modelo cognitivista (ou cognitivo-comportamental) de intervenção ou apenas comportamental.

Desse modo, a primeira parte do artigo pretende expor alguns dos fundamentos e aspectos centrais da proposta de intervenção da ACT. Em seguida, será abordado o que há no modelo da ACT que pode levar à compreensão de que é um modelo cognitivista ou cognitivo-comportamental, explicitando-se, inclusive, como seu fundador concebe a cognição. $\mathrm{O}$ artigo é concluído com algumas considerações finais.

Os pontos a serem abordados, tanto em relação à literatura sobre a ACT (e a Teoria dos Quadros Relacionais) quanto às reflexões da autora, não pretendem aprofundar a discussão de um tema tão complexo (e incômodo para muitos analistas do comportamento), mas apenas iniciar o debate sobre $o$ assunto.

\section{que é ACT?}

A ACT foi fundad, em 1987, pelo norte-americano Steven Hayes e colaboradores (Saban, 2011), e, historicamente, vem sendo incluída no rol das Terapias Cognitivas e Comportamentais da chamada terceira geração (Hayes, 2004; Hayes \& Pistorello, 2011; Higuera, 2006). Uma particularidade das terapias dessa geração, segundo Hayes (2004), consiste no uso mais enfático de estratégias de mudanças contextuais e experienciais, além das estratégias de caráter menos diretivas e didáticas. ${ }^{1}$

O primeiro livro sobre a ACT, intitulado Acceptance and Commitment Therapy: An Experiential Approach to Behavior Change, publicado em 1999, foi escrito por Hayes, Strosahl e Wilson. Na primeira parte da obra, os autores discutem o sofrimento humano, explicitando os efeitos positivos e negativos da linguagem; apresentam os fundamentos teóricos e filosóficos da ACT, tratando do Contextualismo Funcional e do comportamento governado por regras e abordam, ainda, a armadilha do sistema socioverbal discutindo, por exemplo, "razões são causas", "pensamentos e sentimentos são boas razões", "pensamentos e sentimentos são causas" e "deve-se controlar sentimentos e pensamentos" (Hayes, Strosahl \& Wilson, 1999/2003).

Partindo desse cenário, Hayes, Strosahl e Wilson (1999/2003) argumentam que é a forma como o sistema verbal trabalha que leva ao sofrimento e, por isso, a ACT é apresentada como "uma abordagem que segue um modelo de saúde e não de doença" (p. 79).

Em um manual mais recente, Hayes, Strosahl, Bunting, Twohig e Wilson (2010) definiram a ACT" como "uma abordagem funcional contextual de intervenção, baseada na Teoria dos Quadros Relacionais, que vê o sofrimento humano como originário da inflexibilidade psicológica promovida pela esquiva experiencial e fusão cognitiva” (p. 29).

Luciano, Valdivia, Gutiérrez e Páez-Blarrina (2006), além de destacarem a teoria específica que fundamenta a ACT, complementam que esse modelo terapêutico se utiliza da filosofia e de conhecimentos tanto da Análise Aplicada quanto da Análise Experimental do Comportamento.

Especificamente sobre as bases filosóficas da ACT, Hayes (2004) foi explícito quando afirmou que a "ACT é parte da tradição analítica do comportamento e assim está ligada ao Behaviorismo Radical" (p. 646). Por outro lado, por Hayes considerar o termo Behaviorismo Radical "fraco" para

1 Sobre as terapias da terceira geração, sugere-se a leitura de Hayes (2004) e Pérez Álvarez (2006). 
caracterizar a ACT, enfatiza que o Contextualismo Funcional é o que melhor fornece suporte a essa proposta terapêutica. Isso porque se acredita que o Contextualismo Funcional trata de questões presentes na filosofia de Skinner com mais clareza. Tal argumentação é discutível, uma vez que se questiona se o Contextualismo Funcional vai além da filosofia behaviorista radical, como será visto a seguir.

Diversos autores caracterizam o Contextualismo Funcional (Hayes, 2004; Hayes, Strosahl \& Wilson, 1999/2003; Ruiz, 2010; Vilardaga, Hayes, Levin \& Muto, 2009). Contudo, as características apontadas por Luciano et al. (2006) não deixam dúvidas sobre a semelhança com o Behaviorismo de Skinner. Para as autoras, esta filosofia é "monista, não mentalista, não reducionista, ideográfica, e conflui com o Behaviorismo Radical de Skinner e o Interbehaviorismo de Kantor" (p. 180). Mais recentemente, Luciano (2012) chegou a afirmar que "Condutismo es igual al Contextualismo Funcional" e substituir o primeiro termo pelo segundo seria uma forma de esquiva de todas as relações aversivas construídas em torno do primeiro.

Sobre o consenso entre os autores acerca da argumentação supracitada, pode-se dizer que ele não existe. Em um artigo intitulado "Contextual behavioral science: Creating a science more adequate to the challenge of the human condition", Hayes, Barnes-Holmes e Wilson (2012) exploram de forma extensiva aspectos centrais do Contextualismo Funcional (e.g., unidade de análise, ontologia, epistemologia e critério de verdade), parecendo buscar diferenciá-lo do Behaviorismo Radical.

Apesar do esforço dos autores em demarcar perspectivas que seriam características do Contextualismo Funcional quando especificam, por exemplo, que o seu objetivo consiste em "predizer e influenciar, com precisão, alcance e profundidade, organismos inteiros e interagindo no e com um contexto considerado histórica e circunstancialmente" (p. 4), ao longo do texto, as semelhanças com o Behaviorismo de Skinner ainda se mantêm claras.

Dentre as semelhanças observadas entre Behaviorismo Radical e Contextualismo Funcional, a partir do artigo de Hayes, Barnes-Holmes e Wilson (2012), destacam-se: (a) Unidade de análise: para os autores, essa unidade consiste no ato em um contexto (act-in-context), não se podendo separar um do outro. Ao responder com quê o behaviorista radical trabalha, Matos (1997) é enfática ao afirmar que ele trabalha "com o comportar-se dentro de contextos" (p. 46). Além disso, se o comportamento é definido como relação/interação, no Behaviorismo Radical, não se separa o que o organismo faz do contexto no qual o fazer ocorre (cf. Matos, 1997).

(b) Ontologia: os autores ressaltam que experiências privadas são consideradas "como um foco legítimo de análise científica e prática" (p. 5). É possível encontrar, em obras de Skinner, vários trechos que confirmam essa afirmação. Para citar apenas um: "Existe um mundo interno de sentimentos e estados da mente, mas ele está fora do alcance de uma segunda pessoa e, portanto, da ciência. Certamente essa não é uma posição satisfatória. Como as pessoas se sentem é freqüentemente tão importante quanto o que elas fazem" (Skinner, 1989/1991, p. 13 (c) Critério de verdade: segundo os autores, como o critério de verdade é pragmático, os termos "certo", "correto ou "verdadeiro" não se aplicam. No capítulo intitulado "O Behaviorismo Radical como Filosofia da Ciência”, Baum (2005/2006) afirma que essa filosofia está fundamentada no pragmatismo, cabendo ao behaviorista radical buscar descrições do comportamento humano que são úteis, sem qualquer outro compromisso (como buscar a verdade ou o certo, por exemplo).

Feito esse adendo sobre o Contextualismo Funcional, uma vez que a ACT se fundamenta na Teoria dos Quadros Relacionais, antes de caracterizar seu modelo de intervenção, faz-se necessário, mesmo que de forma breve, discorrer sobre a teoria e, por conseguinte, sobre a esquiva experiencial e a fusão cognitiva - conceitos-chave desse modelo.

A Teoria dos Quadros Relacionais (Relational Frame Theory - RFT) tem como foco a linguagem e a cognição (Hayes, 2004; Hayes, Barnes-Holmes \& Roche, 2001; Hayes et al., 2010; Soriano \& Salas, 2006) e parte do pressuposto básico de que humanos estabelecem relações arbitrárias (Ruiz, 2010). 
Na primeira obra sobre a RFT, Hayes, BarnesHolmes e Roche (2001) a definem como "uma abordagem analítico-comportamental para a cognição e a linguagem humana. A RFT trata o responder relacional como um operante generalizado e assim apela para a história de treino com múltiplos exemplares" (p. 141).

Essa teoria se baseia em um conjunto consistente de estudos empíricos (em Blackledge, 2003 e Ruiz, 2010, por exemplo, são citados alguns) e, de acordo com Ruiz (2010) e Soriano e Salas (2006), seria um avanço na área de pesquisas comportamentais, uma vez que "integra áreas diversas da pesquisa comportamental, tais como o seguimento de regras e as relações de equivalência" (Ruiz, 2010, p. 126). Recentemente, inclusive, Abreu e Hübner (2012) reconheceram o esforço dos pesquisadores da RFT em validar, dentro da Análise Experimental do Comportamento, os conceitos por eles utilizados.

Quando se diz que na base dessa teoria está o estabelecimento de relações arbitrárias, isso significa que muito da aprendizagem humana ocorre de forma indireta (cf. Hayes, 1987; cf. Soriano \& Salas, 2006). Por exemplo, se dissermos a uma criança que Bruno é amigo de Jhessica, essa criança derivará uma nova relação, não treinada ou estabelecida de forma indireta, que Jhessica é amiga de Bruno. Do mesmo modo, se dissermos a alguém que bolo é gostoso, e tudo que é gostoso é pouco saudável, essa pessoa poderá derivar uma terceira relação, também indireta, dizendo, então, que bolo (e qualquer outro alimento gostoso) não é saudável. Um último exemplo: se Jhessica se tornou um estímulo aversivo para Bruno, Amanda, que é muito amiga de Jhessica, também pode se tornar um estímulo aversivo para ele, mesmo que nenhuma situação aversiva tenha envolvido Amanda e Bruno.

Os exemplos supracitados ilustram, respectivamente, as principais propriedades do responder relacional ou arbitrário, conforme Hayes (2004), que são o vínculo mútuo (birecionalidade), o vínculo combinatório e a transformação de função. É notória a semelhança entre as duas primeiras propriedades (vínculo mútuo e vínculo combinatório) e as propriedades da equivalência de estímulo, no caso, simetria e transitividade. Entretanto, como vai além da proposta do presente artigo tratar de questões como esta, sugere-se como literatura básica para identificar pontos de convergência e divergência (ou complementaridade) entre as duas teorias o artigo de Moreira, Todorov e Nalini (2006).

Em síntese, sobre a RFT, pode-se dizer que ela

amplia o conhecimento disponível sobre a emergência de novos comportamentos, já que propõe leis que estabelecem as condições para a formação e a alteração de funções, através de procedimentos indiretos, frente aos conhecidos e bem estabelecidos procedimentos diretos de manejo de contingências para o estabelecimento e mudança de funções reforçadoras, aversivas, motivacionais, e discriminativas de aproximação e evitação. (Soriano \& Salas, 2006, p. 82.

Passando, a partir deste ponto, a enfocar os conceitos-chave da intervenção na ACT, evitação ou esquiva experiencial e fusão cognitiva, pode-se afirmar que a primeira consiste na tentativa de evitar ou de exercer controle sobre os eventos psicológicos (ou privados, como denominados por Skinner ${ }^{2}$ ) em si (Hayes et al., 2010; Luoma, Hayes \& Walser, 2007). Se uma pessoa se sente ansiosa quando precisa ir ao centro da cidade, ela pode se esquivar da própria ansiedade que ocorre nessa situação e não somente da situação. Sobre a possibilidade de uma emoção ou sentimento se tornar um segundo estímulo aversivo condicionado, Skinner (1989/1991) já a havia reconhecido. Cabe ainda destacar que a esquiva experiencial abrange não apenas a esquiva emocional (de sentir raiva, de sentir inveja), mas a esquiva de qualquer evento psicológico, como pensamentos, recordações, dentre outros (Hayes, 1987; Luoma et al., 2007).

Já a fusão cognitiva (que pode ser compreendida como a fusão ao pensamento ou às palavras) seria a tendência do ser humano a interagir com o mundo a partir das funções verbais que lhe foram atribuídas (e.g., bom, ruim, feio, melhor), distanciando-se dos eventos propriamente ditos (Hayes et al., 2010; Luoma et al., 2007). Um indivíduo que diz

2 Ao longo do artigo, o termo evento psicológico será utilizado em substituição a evento privado, dados os problemas com esta última nomenclatura, conforme apontou Tourinho (2009). 
a si mesmo que não existem pessoas boas no mundo e passa a se isolar, está respondendo ao pensamento como se fosse o próprio evento. Ele não discrimina que se tratam de dois eventos distintos (o pensamento e "a maldade das pessoas"). O efeito da fusão ao pensamento seria um repertório limitado por manter o indivíduo afastado do mundo das situações externas que o cercam e extremamente sensível ao seu mundo psicológico (Soriano, 2010). Nas palavras de Soriano, "É ir sem direção, cegados pelas palavras como 'não posso', 'estou triste', 'me sufoco', (. . .) É enganar-se pela ilusão verbal, é ver as árvores sem ver o bosque" (p. 7).

Acerca da intervenção propriamente dita, Hayes (2004) argumenta que esse processo visa a identificar, constantemente, tanto a fusão ao pensamento quanto a esquiva experiencial a fim de que o cliente mude conforme os seus valores, desenvolvendo padrões comportamentais cada vez mais amplos e efetivos.

Como objetivos específicos do modelo de intervenção da ACT, Hayes, Strosahl e Wilson (1999/2003) apontam o aceitar, o escolher e o agir. Por aceitação, entende-se uma ação ativa e consciente de experienciar todo e qualquer evento psicológico, sem julgá-lo (Hayes, 2004; Luoma et al., 2007). Significa tratar "sentimentos como sentimentos; (. . .) pensamentos como pensamentos; (. . .) sensações como sensações" (Hayes, 2004, p. 656). Ou ainda, é abandonar a luta pelo controle dos eventos psicológicos. A escolha implica decidir fazer algo com o que ocorre no presente, já que não se pode alterar a história passada de um indivíduo (Saban, 2011). Por fim, a ação seria o comprometer-se com as mudanças possíveis, de acordo com o que cada um valoriza (Luoma et al., 2007; Saban, 2011).

Considerando que os obstáculos para mover-se em direção aos valores pessoais são a fusão ao pensamento e a esquiva experiencial (originários, sobretudo, do campo verbal), segundo Hayes, Strosahl e Wilson (1999/2003), a ACT objetiva diminuir os efeitos da linguagem literal, observada quando se responde a um evento verbal como se ele fosse o próprio evento que se descreve. Por exemplo, reagir à palavra câncer como se estivesse diante de um paciente com câncer (sentindo pena, medo, dentre outros).
Com base na literatura mais atual, pode-se dizer que os objetivos ou processos de aceitação, escolha e ação, em última instância, visam a desenvolver no cliente a flexibilidade psicológica (Luoma et al., 2007; Saban, 2011), definida como a habilidade de permanecer em contato com o presente e alterar ou manter-se na situação, quando é importante, para alcançar os valores pessoais (Hayes et al., 2010).

As características do modelo ACT são sintetizadas por Luciano et al. (2006) da seguinte forma:

(a) tratamento centrado nas ações valorizadas pelo cliente; (b) considera o mal-estar/sofrimento como natural, produto da condição humana como seres verbais; (c) define que se aprende a resistir ao sofrimento normal e é justamente essa resistência que gera o sofrimento patológico; (d) promove a análise funcional dos comportamentos do paciente (...); (e) tem por objetivo flexibilizar a reação ao mal-estar (. . .); (f) implica clarificar valores para atuar na direção que se valoriza, aceitando com plena consciência os eventos privados que surge, e praticar a aceitação deles .... (pp. 192-193)

Segundo o próprio Hayes (2004), a ACT inclui intervenções tanto comportamentais quanto cognitivas. Mais recentemente, Hayes e Pistorello (2011) afirmaram que a ACT "Baseia-se no melhor dos dois campos: terapia comportamental/análise do comportamento e Terapia Comportamental e Cognitiva" (Prefácio). Embora se possa questionar se, de fato, a ACT é o melhor modelo de intervenção comportamental e/ou cognitivista, como costumam afirmar alguns autores, diante do que foi apresentado até aqui é que será abordado, no tópico seguinte, o que há de cognitivo no modelo da ACT.

\section{que há de "cognitivo" na}

\section{proposta da ACT?}

A resposta a esse questionamento, de certo modo, parece fácil e simples, como se pode ver em trechos anteriores deste artigo, mas seguramente ela não é consensual. Higuera (2006), por exemplo, argumentou que "A relação entre a terapia de aceitação e compromisso e a terapia cognitivo-comportamental levanta polêmicas com grandes desencontros, sobretudo em função de desconhecimentos e sim- 
plificações mútuas" (p. 288). Em outro momento do mesmo artigo, o autor chega a afirmar que as terapias cognitivas (ou os terapeutas cognitivos) encontrarão problemas ao tentar integrar a ACT à sua atuação.

Sobre a possibilidade (ou impossibilidade) de integrar a ACT aos modelos cognitivo ou cognitivo-comportamentais de atuação, em 2005, o Journal of Rational-Emotive \& Cognitive-Behavior Therapy (Vol. 23, No. 2) publicou um conjunto de artigos que discutem o assunto tendo como modelo cognitivo-comportamental a Terapia Racional Emotiva Comportamental, de Albert Ellis. O número inclui artigos dos próprios proponentes dos referidos modelos, Hayes e Ellis.

De fato, Hayes (1987) aponta para a compatibilidade entre a ACT e alguns aspectos de certos modelos cognitivistas, como o de Beck, bem como usa o termo "cognição" em todos os seus artigos e livros, sendo enfático ao afirmar que a RFT consiste em uma teoria da cognição. Acredita-se, desse modo, que são esses, possivelmente, os fatores que contribuem para que se inclua a ACT no conjunto das intervenções cognitivistas ou cognitivo-comportamentais. Contudo, a concepção de Hayes acerca da cognição "parece" muito distante daquela que é dada pelos cognitivistas (cognição como evento causal, e não comportamental).

Ainda na década de 1980, Hayes (1987) discorreu sobre como compreende o pensamento - termo usado neste artigo como sinônimo de cognição, embora propostas cognitivistas os diferenciem (Costa, 2002).

Hayes (1987) concebeu o pensamento como um evento privado, assim como Skinner. Nesse mesmo texto de 1987, o autor assim se posiciona: "pensamentos não são substancialmente diferentes em virtude de sua natureza privada. Eles podem ter propriedades especiais porque são verbais, mas eles ainda são comportamentos". (p.2) Ampliando um pouco mais, os eventos psicológicos, de forma geral, são entendidos, no contexto da ACT, como um "conjunto de interações em curso entre organismos completos e contextos definidos circunstancialmente e historicamente" (Hayes, 2004, p. 646). Essa compreensão em nada se distancia ou fere a proposição que Skinner desenvolveu ao longo de sua obra, nem as proposições contemporâneas sobre o assun- to, como a de Tourinho (2009) - que trata os fenômenos psicológicos como relações comportamentais, envolvendo diferentes níveis de complexidade (filogenético, ontogenético e cultura) -, as quais incluem, necessariamente, dimensões públicas.

É com essa concepção de pensamento que Hayes (1987) passa a abordar o chamado controle cognitivo como uma relação comportamento-comportamento. Desse modo, o controle cognitivo seria um tipo de controle verbal, o qual envolve a análise de contingências, cabendo ao analista do comportamento investigar, então, "Quais são as contingências que dão suporte à relação entre pensamentos e outras formas de ação humana. Neste ponto de vista, os pensamentos não produzem necessariamente nenhum efeito em outros comportamentos" (Hayes, 1987, p. 3). Um exemplo prático seria entender o que, na história de uma pessoa, faz com que o pensamento "sou uma pessoa burra" controle, por exemplo, o comportamento autolesivo de bater a cabeça na parede.

Ao demarcar a diferença da sua proposição de pensamento (ou cognição) para o que denomina de posição cognitivista tradicional, Hayes (1987) inclusive aproxima a posição cognitivista ao raciocínio estímulo-resposta, já que "a resposta aberta é automática e diretamente produzida pelo pensamento" (p. 6). Outra crítica explícita à proposição cognitivista de enfatizar os pensamentos é encontrada em Hayes e Wilson (1995), quando afirmam, ao se referirem à depressão, que, comumente, a alteração da topografia e da frequência de eventos cognitivos, não necessariamente precede mudanças em padrões de comportamento. É por esse motivo que a ACT busca alterar a função do pensamento e não a sua frequência e/ou conteúdo (Hayes, 2004; Luciano et al., 2006). Assim, se a ACT se utiliza de intervenções cognitivas, de fato, como declaram Hayes (2004) e Hayes e Pistorello (2011), por exemplo, tais intervenções possuem objetivos marcadamente distintos.

O que talvez Hayes tenha pretendido enfatizar, que difere da interpretação skinneriana (embora mantenha a consistência com um recorte relacional, contextual e pragmático presentes na filosofia de Skinner), foi que tanto a linguagem como a cognição (ambas entendidas como comportamento) fazem parte de quadros relacionais e se torna im- 
prescindível entendê-las para explicar parte significativa do comportamento humano. "Quando nós pensamos, raciocinamos, falamos com significado ou ouvimos com compreensão, nós fazemos isto por derivar relações entre eventos - entre palavras e eventos, palavras e palavras, eventos e eventos" (Hayes, 2004, p. 649).

Havendo ou não concordância com a teoria elaborada por Hayes, ou ainda sobre a real necessidade da criação de uma "nova" teoria, não parece difícil concordar com a afirmação de Hayes e Wilson (1995) de que a RFT trata da cognição como um evento comportamental e não causal. Nas palavras de Higuera (2006), "A teoria dos quadros relacionais permite uma abordagem do processo do pensamento a partir de uma perspectiva do behaviorismo radical, isto é, aquela que considera os pensamentos como comportamentos" (p. 298).

\section{Considerações Finais}

Buscou-se, com este artigo, discutir se a ACT se enquadra em um modelo cognitivista/cognitivo-comportamental (que atribum status de mediadores causais às cognições ou pensamentos e, por este motivo, elegem como foco de intervenção tais eventos) ou apenas comportamental. Diante do exposto, é possível concluir que há duas respostas possíveis: sim, porque usa o termo "cognição" e compreende que tal conceito é essencial para entender e intervir em problemas clínicos; e não, porque as cognições não são vistas como variáveis independentes, como propõem muitos (ou todos) os modelos cognitivistas. Tais respostas se sustentam na argumentação de Hayes, Strosahl e Wilson (1999/2003) quando dizem que a ACT é uma forma de terapia comportamental, "Mas o conteúdo dessa teoria é tudo sobre cognição e emoção, mesmo que o modelo não seja cognitivo no sentido de processamento de informações. Assim, é razoável chamá-la de terapia cognitivo-comportamental" (p. 79).

Como exposto, a ACT consiste em um modelo terapêutico que se utiliza da RFT e da filosofia do Contextualismo Funcional (e/ou do Behaviorismo Radical) e que, mesmo dando ênfase à cognição (ou pensamento), distancia-se de modelos que concebem esse fenômeno como causal.

Apesar de observar, no contato com alguns behavioristas radicais, que a ACT vem sendo tra- tada como um modelo cognitivista, questionando-se sua coerência e/ou consistência com a Análise do Comportamento (como pontua Tourinho, 2011, por exemplo), acredita-se que o que foi abordado, a partir das referências consultadas, evidencia que essa proposta de intervenção se mantém fiel à interpretação skinneriana de eventos psicológicos, tratando as cognições/pensamentos como variáveis dependentes.

Convém ponderar que, dentre os que escrevem e aplicam a ACT, existem aqueles que são mais e outros menos fiéis aos princípios da Análise do Comportamento, assim como ocorre com a prática denominada no Brasil de Terapia Analíticocomportamental.

Alguns (ou muitos) incômodos podem advir da literatura da ACT, sobretudo nos contatos iniciais este artigo é, aliás, produto de alguns deles. Hayes e seus colaboradores usam o termo "cognição" e mesmo "mente" em suas publicações (algo que um behaviorista radical, no mínimo, evita); propõem interpretações incomuns como separar o "eu" do comportamento e ainda enfatizam, na intervenção, práticas orientais, como o mindfulness. Esses aspectos, dentre outros, aliados à figura polêmica de Hayes, podem causar confusão e aversão aos que escutam pela primeira vez a proposta - como muitos dos alunos da graduação com os quais a autora tem contato, e, inclusive, com ela própria.

Sem entrar no mérito de um possível apelo popular ligado à ACT e/ou à figura do seu fundador e da leitura ainda incipiente da autora (uma behaviorista radical convicta) acerca da ACT e da RFT, a proposta de intervenção da ACT, até o momento, não se mostrou em nada incompatível com os fundamentos da Terapia Analítico-comportamental. A diferença fundamental talvez esteja na ênfase dada pela ACT para à relação entre linguagem (comportamento verbal) e cognição (pensamento como resposta encoberta) como fundamental no contexto clínico.

O debate está aberto. 


\section{Referências}

Abreu, P. R., \& Hübner, M. M. C. (2012). O comportamento verbal para B. F. Skinner e para S. C. Hayes: Uma síntese com base na mediação social arbitrária do reforçamento. ACTA Comportamentalia, 2), 367-381.

Barbosa, J. I. C., \& Borba, A. (2010). O surgimento das terapias cognitivo-comportamentais e suas consequências para o desenvolvimento de uma abordagem clínica analítico-comportamental dos eventos privados. Revista Brasileira de Terapia Comportamental e Cognitiva, XI), 6079.

Baum, W. M. (2006). Compreender o behaviorismo: Ciência, comportamento e cultura (2a. ed.). Porto AlegrS: Aremed. (Trabalho original publicado em 2005

Blackledge, J. T. (2003). An introduction to relational frame theory: Basics and applications. The Behavior Analyst Today, ), 421-433.

Costa, N. (2002). Terapia analítico-comportamental: Dos fundamentos filosóficos à relação com o modelo cognitivista. Santo AndrP: ESETes.

Forsyth, J. P., Lejuez, W., Hawkins, R. P., \& Eifert, G. H. (1996). Cognitive vs.Ccontextual causation: Different world views but perhaps not irreconcilable. Journal of Behavior Therapy and Experimental Psychiatrt, 2), 369-376.

Guilhardi, H. J., \& Queiroz, P, B. P. S. (s.d). As bromélias e a teia de aranha. Disponível em http:// www.terapiaporcontingencias.com.br/pdf/helio/Bromelia_aranha.pdf

Hayes, S. C. (1987). Um enfoque contextual para mudança terapêutica. Disponível em http:// www.oocities.org/br/estagioildenor/textos.html

Hayes, S. (2004). Relational frame theory, and the third wave of behavioral and cognitive therapies. Behavior Therapy, 35, 639-665.

Hayes, S. C., Barnes-Holmes, D., \& Roche, B. (2001). Relational frame theory: A post skinnerian account of human language and cognition. New York: Kluwer Academic/Plenum

Hayes, S. C. Barnes-Holmes, D., \& Wilson, K. G. (2012). Contextual behavioral science: Creating a science more adequate to the challenge of the human condition. Journal of Contextual Behavioral Sciences, 1, 1-16.
Hayes, S. C., \& Pistorello, J. (2011). Prefácio. Em M. T. Saban. Introdução à terapia de aceitação e compromisso. Santo André: ESETes.

Hayes, S. C., Strosahl, K. D., Bunting, K., Twohig, M., \& Wilson, K. G. (2010). What is acceptance and commitment therapy? Em S. C. Hayes, \& K. D. Strosah. sOrgs.), A practical guide to acceptance and commitment therapy (pp. 1-29). New York: Springer.

Hayes, S. C., Strosahl, K. D., \& Wilson, K. G. (2003). Acceptance and commitment therapy: An experimental approach to behavior change. New York/ London: The Guilford Press. (Trabalho original publicado em 1999

Hayes, S. C., \& Wilson, K. G. (1995). The role of cognition in complex human behavior: A contextualistic perspective. Journal of Behavior Therapy and Experimental Psychiatrt, 26, 241248.

Hawkins, R. M. F. (1996). What are the benefits from a debate about cognition, behavior, and causality? Journal of Behavior Therapy and Experimental Psychiatrt, 2), 351-356.

Higuera, J. A. G. (2006). La terapia de aceptación y compromiso (ACT) como desarrollo de la terapia cognitivo conductual. EduPsykhé, ), 287304.

Kohlenberg, R. J., Tsai, M., Kanter, J. W., \& Parker, C. R. (2011). Self e mindfulness. Em M. Tsai, R. J. Kohlenberg, J. W. Kanter, B. Kohlenberg, W. C. Follette \& G. M. Callaghan (Orgs.). Um guia para a psicoterapia analítica funcional: Consciência, coragem, amor e behaviorismo (XYZ, Trad.; pp. 139-170). Santo AndrP: ESEtec.

Luciano, M. C. (2012)

Luciano, M. C., Valdivia, S., Gutiérrez, O., \& PáezBlarrina, M. (2006). Avances desde la terapia de aceptación y compromiso (ACT). EduPsykhé, , 173-201.

Luoma, J. B., Hayes, S. C., \& Walser, R. D. (2007). The six core process of ACT and their common target.nEm AUTORES Learning ACT: An acceptance \& commitment therapy skills-training manual for therapist (pp. 9-22).ACIDADE: New Harbinger Publications.

Matos, M. A. (1997). Com o quê o behaviorista radical trabalha. Em R. A. Banaco (Org.). Sobre 
comportamento e cognição: Vol. 1. Aspectos teóricos, metodológicos e de formação em análise do comportamento e terapia cognitivista (pp. 4553). Santo AndrP: ESETes.

Moreira, M. B., Todorov, J. C., \& Nalini, J. E. G. (2006). Algumas considerações sobre o responder relacional. Revista Brasileira de Terapia Comportamental e Cognitiva, VII), 192-211.

Pérez Alvarez, M. (2006). La terapia de conducta de tercera generación. EduPsykhé, ), 159-172.

Roemer, L., \& Orsillo, S. M. (2010). Introdução. Em XYZ (Org.), A prática da terapia cognitivo-comportamental baseada em mindfulness e aceitação (M. A. V. Veronese, Trad.;(pp. 18-32). Porto AlegrS: Artmed (Trabalho original publicado em 2009

Ruiz, F. J. (2010). A review of acceptance and commitment therapy (ACT) empirical evidence: Correlational, experimental psychopathology, component and outcome studies. International Journal of Psychology and Psychological Therapy, 1), 125-162.

Saban, M. T. (2011). Introdução à terapia de aceitação e compromisso. Santo André: ESETes.

Skinner, B. F. (1991). Questões recentes na análise comportamental. (A. L. Nero, Trad.). CampinaP: Papirus. (Trabalho original publicado em 1989

Soriano, C. L. (2012, novembro). ACT Acceptance and commitment therapy. Curso promovido pelo Núcleo Paradigma: Análise do Comportamento, São Paulo, SP.

Soriano, C. L. (2010). Condición humana y felicidad: Hechos y palabras. Dispon;ivel em http://cms. ual.es/idc/groups/public/@orgob/@gabcomunicacion/documents/documento/01 oct2010_ discurdo_rector_apertura_pdf.pdf

Soriano, M. C. L., \& Salas, M. S. V. (2006). La terapia de aceptación y compromiso (ACT): Fundamentos, características y evidencia. Papeles del Psicólogo, 2), 79-91.

Tourinho, E. Z. (2009). Subjetividade e relações comportamentai). São PaulP: Paradigma.

Tourinho, E. Z. (2011). Notas sobre o behaviorismo de ontem e de hoje. Psicologia: Reflexão e Crítica, 2), 186-194.
Vilardaga, R., Hayes, S. C., Levin, M. E., \& Muto, T. (2009). Creating a strategy for progress: a contextual behavioral science approach. The Behavior Analyst, 32, 105-133.

\section{Informações do Artigo}

Histórico do artigo:

Submetido em: 18/06/2013

Primeira decisão editorial: 15/08/2013

Segunda decisão editorial: 26/08/2013

Aceito em: 03/09/2013 